\title{
A RESULT ON THE SET-THEORETIC COMPLETE INTERSECTION PROBLEM
}

\author{
T. T. $\mathrm{MOH}^{1}$
}

ABSTRACT. This article establishes a certain arithmetical criterion for settheoretic complete intersection for irreducible analytic space curves.

Introduction. The object of this article is to study the set-theoretic complete intersection problem for the analytic space curves over an algebraically closed field $k$ of characteristic 0 . Let $\tau$ be a map from the power series ring of three variables $k[[x, y, z]]$ to $k[[t]]$ with

$$
\tau(x)=t^{l}+\ldots, \quad \tau(y)=t^{m}+\ldots, \quad \tau(z)=t^{n}+\ldots .
$$

Let $P$ be the kernel of the map $\tau$. The problem is to investigate if $P=\sqrt{(f, g)}$ for some suitable $f$ and $g$. Note that an automorphism in $k[[x, y, z]]$ will reduce $\tau(x), \tau(y)$ and $\tau(z)$ to polynomials in $t$. Thus an affirmative answer to the settheoretic complete intersection for the algebraic space curves will imply an affirmative answer to the present problem, while a solution to the present problem will certainly throw light to the global problem of the algebraic space curves. Our result is Theorem 1 which gives some partial solution to the analytic problem.

THEOREM 1. If $l<m,(l, m)=1$ and $(l-2) m<n$, then the ideal $P$ is a set-theoretic complete intersection.

PROOF. Note that the conductor $\left(t^{c^{*}}\right)$ of $k[[t]]$ over $k[[\tau(x), \tau(y)]]$ is with $c^{*}=$ $(l-1)(m-1)<(l-1) m$. Thus if $(l-1) m \leq n$ then $P$ defines a plane curve. We may henceforth assume that $n \notin$ the semigroup generated by $l$ and $m$ and the following: $(l-2) m<n<(l-1) m$. We may choose $t$ properly with

$$
\tau(x)=t^{l}, \quad \tau(y)=t^{m}+\ldots, \quad \tau(z)=t^{n}+\ldots
$$

We shall view $(\tau(y), \tau(z))$ as a point $P_{l}$ in the affine plane over an algebraic closure of $k[[t]]$ (i.e., the Puiseux field). Let $\omega$ be an $l$ th primitive root of unity. The following conjugations

$$
t \rightarrow \omega^{i} t, \quad i=1, \ldots, l,
$$

will produce $l$ points $P_{1}, \ldots, P_{l}$ in the affine plane. We shall construct a polynomial $f(y, z)$ of the following form over $k[[t]]$

$$
f(y, z)=y^{l-1}+\alpha_{1} z+\alpha_{2} y^{l-2}+\cdots+\alpha_{l-1}+\alpha_{l}
$$

with $f\left(P_{i}\right)=0$ for $i=1, \ldots, l$.

Received by the editors March 25, 1980 and, in revised form, October 14, 1981.

1980 Mathematics Subject Classification. Primary 14M10.

Key words and phrases. Set-theoretic complete intersection, analytic curves, semigroups.

${ }^{1}$ This work was partially supported by NSF at Purdue University. 
First note that if for $0 \leq i<(l-1)$ we have $n \equiv i m \bmod l$ then we have for some positive $s, n=i m+s l$. Hence $n \in$ the semigroup generated by $l$ and $m$. We must then have

$$
n \equiv(l-1) m \bmod l \text {. }
$$

Let $\alpha_{1}=t^{(l-1) m-n} \alpha_{1}^{*}$ and $\alpha_{i}=t^{(i-1) m} \alpha_{i}^{*}$ for $i=2, \ldots, l$. The conditions $f\left(P_{i}\right)=0$ for $i=1, \ldots, l$ are linear equations in the indeterminates $\alpha_{1}^{*}$. Let us factor out the common factor $t^{(l-1) m}$ from these equations. Then the matrix of the leading coefficients is the following nonsingular matrix

$$
\left[\begin{array}{lll}
\left(\omega^{m}\right)^{l-1}, & \left(\omega^{m}\right)^{l-2}, & \ldots, 1 \\
\left(\omega^{2 m}\right)^{l-1}, & \left(\omega^{2 m}\right)^{l-2}, & \ldots, 1 \\
& & \\
1, & 1, & \ldots, 1
\end{array}\right]
$$

Thus there exist solutions for $\alpha_{1}^{*}, \ldots, \alpha_{l}^{*}$ in $k[[t]]$. Similarly, we may construct a polynomial $g(y, z)$ of the following form over $k[[t]]$

$$
g(y, z)=z+\beta_{2} y^{l-2}+\cdots+\beta_{l}
$$

with $g\left(P_{i}\right)=0, i=2, \ldots, l$.

Let $F(y, z)=\Pi$ (all conjugates of $f(y, z)), G(y, z)=\Pi$ (all conjugates of $g(y, z))$. Then clearly $F, G \in P \subset k[[x, y, z]]$. We shall prove that $\sqrt{(F, G)}=P$.

It follows from the forms of $F, G$ that

$$
F=y^{(l-1) l} \bmod x, \quad G=z^{l} \bmod x .
$$

Thus we conclude that the variety $V$ defined by $(F, G)$ contains no plane curve in the $y z$ plane defined by $(x)$. Let $W$ be any irreducible curve in the variety $V$. The parameterization of $W$ can be taken as follows with fractional exponents allowed

$$
\tau^{*}(x)=t^{l}, \quad \tau^{*}(y)=r_{2} t^{m^{*}}+\ldots, \quad \tau^{*}(z)=r_{3} t^{n^{*}}+\ldots
$$

Then $\left(\tau^{*}(y), \tau^{*}(z)\right)$ can be considered as a point in the affine plane over the algebraic closure of $k[[t]]$. In other words $\left(\tau^{*}(y), \tau^{*}(z)\right) \in \mathcal{V}$ (a conjugate of $\left.f\right) \cap \mathcal{V}$ (a conjugate of $g$ ). However, it is easy to see these two curves intersect at only $(l-1)$ points of $\left\{P_{1}, \ldots, P_{l}\right\}$. Thus $\left(\tau^{*}(y), \tau^{*}(z)\right)$ is a conjugate of $(\tau(y), \tau(z))$. Clearly we have $\sqrt{(F, G)}=p . \quad$ Q.E.D.

EXAMPLE. If $l=3$, then $P$ is a set-theoretic complete intersection.

\section{REFERENCES}

1. R. C. Cowsik and M. V. Nori, Affine curves in char $P$ are set-theoretic c.i., Invent. Math. 45 (1978).

2. D. Ferrand, Les modules projectifs de type fini, Sém. N. Bourbaki, no. 484 (1976).

3. J. Herzog, Generators and relations of Abelian semigroups and semigroup rings, Manuscripta Math. 3 (1970).

4. T. T. Moh, On the unboundedness of generators of prime ideals, J. Math. Soc. Japan 26 (1974). 